\title{
FAIR SEMIGROUPS AND MORITA EQUIVALENCE
}

\author{
VALDIS LAAN AND LÁSZLÓ MÁRKI
}

\begin{abstract}
In analogy to the xst-rings studied by García and Marín, we define fair semigroups and investigate Morita equivalence for a subclass of them. In particular, we present examples for semigroups which are Morita equivalent but not strongly Morita equivalent.
\end{abstract}

\section{INTRODUCTION}

In recent years, a satisfactory theory of Morita equivalence has been developed for semigroups with local units, see [6-8]. The efficiency of this theory is largely due to the fact, established by Lawson [8], that in this case Morita equivalence is strong in the sense that it comes from a well-behaved Morita context. Little is known about Morita equivalence under assumptions weaker than having local units, see [6]. In particular, no example has been known for Morita equivalence between two semigroups which are not strongly Morita equivalent.

In the present paper we consider Morita equivalence in a different class of semigroups, which we call fair semigroups. This class corresponds to the class of xstrings considered by García and Marín [3], based on previous work by Xu, Shum, and Turner-Smith [11], and the tools we use also correspond to those in [3]. Every semigroup $S$ has a largest unitary right ideal $U\left(S_{S}\right)$, and if $S$ is right fair then $U\left(S_{S}\right)$ is a two-sided ideal. We show that if $S$ and $T$ are right fair semigroups such that $U\left(S_{S}\right)$ and $U\left(T_{T}\right)$ have common weak right local units then $S$ and $T$ are Morita equivalent if and only if $U\left(S_{S}\right)$ and $U\left(T_{T}\right)$ are also. This makes it possible to handle cases where one of the semigroups is not factorisable, and we obtain semigroups $S, T$ which are Morita equivalent but not strongly Morita equivalent.

A semigroup $S$ is called factorisable if every element of $S$ is a product of two elements. We say that an element $s$ of a semigroup has a weak right local unit $u$ if $s u=s$. Weak left local units are defined similarly. A semigroup has weak local units if each of its elements has both a weak right and a weak left local unit, and local units if the elements $u$ above can always be chosen to be idempotent.

We say that a semigroup $S$ has common weak right local units if for every $s, t \in S$ there exists $u \in S$ such that $s=s u$ and $t=t u$. Semigroups with common weak left local units are defined similarly. A semigroup has common weak local units if it has common weak right local units and common weak left local units.

2010 Mathematics Subject Classification. 20M10, 20M30.

Key words and phrases. Fair semigroup, unitary act, Morita equivalence.

Research of the first named author was partially supported by the Estonian Institutional Research Project IUT20-57. Research of the second named author was partially supported by the Hungarian National Research Foundation grant no. K101515. Mutual visits of the authors were made possible by the exchange agreement between the Estonian and the Hungarian Academies of Sciences. 
Clearly, if $S$ is a semigroup with common weak right local units then for every finite subset $\left\{s_{1}, \ldots, s_{n}\right\} \subseteq S$ there exists $u \in S$ such that $s_{k}=s_{k} u$ for all $k \in\{1, \ldots, n\}$.

A semigroup $S$ is called right reductive if, for every $s, t \in S, s z=t z$ for all $z \in S$ implies $s=t$. For a semigroup $S$ we have the following implication:

common weak right local units $\Rightarrow$ right reductive.

Indeed, let $s, t \in S$, where $S$ has common weak right local units. Then $s=s u$ and $t=t u$ for some $u$ in $S$. Suppose that $s z=t z$ for all $z \in S$. In particular, $s u=t u$, which means that $s=t$. Hence $S$ is right reductive.

\section{FAIR SEMIGROUPS}

In this section we introduce and investigate fair semigroups; these are semigroups which correspond to the xst-rings investigated by García and Marín [3].

Definition 1. Let $S$ be a semigroup. A right $S$-act $A_{S}$ is called

(1) unitary if $A S=A$;

(2) s-unital if for every $a \in A$ there exists $s \in S$ such that $a s=a$.

Clearly, every s-unital right act is unitary.

Definition 2. We say that a semigroup $S$ is a right fair semigroup if every subact of a unitary right $S$-act is unitary. Dually one defines left fair semigroups. By a fair semigroup we mean a semigroup which is both left and right fair.

Proposition 1. A semigroup $S$ is a right fair semigroup if and only if every unitary right $S$-act is s-unital.

Proof. Necessity. Let $S$ be a right fair semigroup and let $A_{S}$ be a unitary act. Then, for every $a \in A$, the subact $a S^{1} \subseteq A_{S}$ is unitary. But this implies that $A_{S}$ is s-unital.

Sufficiency is evident.

Proposition 2. A semigroup $S$ has weak right local units if and only if $S$ is a factorisable right fair semigroup.

Proof. Necessity. Assume that a semigroup $S$ has weak right local units. Then it is clearly factorisable. Let $A_{S}$ be unitary and $a \in A$. Then $a=a^{\prime} s$ for some $a^{\prime} \in A$ and $s \in S$. By assumption, $s=s u$ for some $u \in S$. Hence $a=a^{\prime} s=a^{\prime} s u=a u$. This proves that $S$ is a right fair semigroup.

Sufficiency. Let $S$ be a factorisable right fair semigroup. Since $S$ is factorisable, the right $S$-act $S_{S}$ is unitary, hence for each $s \in S$ there exists $u \in S$ such that $s=s u$.

Example 1. Factorisable semigroups need not have weak right local units. Take the multiplicative semigroup of real numbers between 0 and 1 ( 0 and 1 excluded). This semigroup is factorisable but none of its elements has a weak right local unit.

There are also fair semigroups which do not have weak right local units (see Example 3(4)).

Next we give a description of fair semigroups which does not involve $S$-acts.

Theorem 1. A semigroup $S$ is a right fair semigroup if and only if for every sequence $\left(s_{i}\right)_{i \in \mathbb{N}} \in S^{\mathbb{N}}$ of elements of $S$ there exists $n \in \mathbb{N}$ such that the product $s_{n} \ldots s_{1}$ has a weak right unit. 
Proof. Necessity. Let $S$ be a right fair semigroup. Consider a sequence $\left(s_{i}\right)_{i \in \mathbb{N}} \in$ $S^{\mathbb{N}}$. We repeat a construction from [5]. Take the right $S$-act

$$
F_{S}:=\coprod_{\mathbb{N}} S=\bigcup_{n \in \mathbb{N}}(\{n\} \times S)
$$

with the right $S$-action $(n, s) z:=(n, s z)$, and put

$$
M_{S}:=F / \sim,
$$

where the right $S$-act congruence $\sim$ on $F$ is defined by

$$
(k, s) \sim(l, z) \Longleftrightarrow(\exists n \in \mathbb{N})\left(n \geq k, l \text { and } s_{n} \ldots s_{k+1} s=s_{n} \ldots s_{l+1} z\right),
$$

$k, l \in \mathbb{N}, s, z \in S$ (for $n=k, s_{n} \ldots s_{k+1} s:=s$ ). Denote the congruence class of a pair $(k, s)$ by $[k, s]$. Take any $[k, s] \in M$, where $k \in \mathbb{N}, s \in S$. Since $\left(s_{k+2} s_{k+1}\right) s=$ $s_{k+2}\left(s_{k+1} s\right)$, we have $(k, s) \sim\left(k+1, s_{k+1} s\right)$, and hence $[k, s]=\left[k+1, s_{k+1} s\right]=$ $\left[k+1, s_{k+1}\right] s \in M S$. Thus $M_{S}$ is unitary.

By assumption, $M_{S}$ is s-unital. Take the element $\left[1, s_{1}\right] \in M$. Then $\left[1, s_{1}\right]=$ $\left[1, s_{1}\right] u=\left[1, s_{1} u\right]$ for some $u \in S$. Hence there exists $n \in \mathbb{N}$ such that $s_{n} \ldots s_{2} s_{1}=$ $s_{n} \ldots s_{2} s_{1} u$, so $u$ is a weak right unit for $s_{n} \ldots s_{2} s_{1}$.

Sufficiency. Let $A_{S}$ be a unitary $S$-act and $a_{0} \in S$. Repeatedly using unitariness we can find elements $a_{1}, a_{2}, \ldots \in A$ and $s_{1}, s_{2}, \ldots \in S$ such that $a_{i-1}=$ $a_{i} s_{i}$ for each $i \in \mathbb{N}$. By assumption there exists $n \in \mathbb{N}$ such that $s_{n} \ldots s_{1}$ has a weak right unit, say $u$. Hence

$$
a_{0}=a_{1} s_{1}=a_{2} s_{2} s_{1}=\ldots=a_{n} s_{n} \ldots s_{1}=a_{n} s_{n} \ldots s_{1} u=a_{0} u .
$$

Thus $S$ is a right fair semigroup.

Example 2. Consider any set $S$ which contains at least three elements. Choose distinct elements $0, a, e \in S$ and define a multiplication on $S$ as follows. Put $s e:=s$ for every $s \in S$ and let all other products be 0 . In the smallest case when $S=\{0, a, e\}$, the multiplication table is thus

\begin{tabular}{l|lll} 
& 0 & $a$ & $e$ \\
\hline 0 & 0 & 0 & 0 \\
$a$ & 0 & 0 & $a$ \\
$e$ & 0 & 0 & $e$
\end{tabular}.

If $x, y, z \in S$ and $z \neq e$ then $(x y) z=0=x(y z)$. If $z=e$ then $(x y) z=x y=x(y z)$. Hence $S$ is a semigroup. The element $e$ is a right identity of $S$; in particular, $S$ is factorisable and right fair. On the other hand, $S$ is not left fair because the subproducts of the sequence $a, e, e, e, \ldots$ are all equal to $a$ and $s a=0$ for all $s \in S$.

Note also that the elements $a$ and $e$ have no common weak left unit, hence a (finite) factorisable semigroup need not have common weak left (or, dually, right) local units.

Let us list some consequences of Theorem 1 .

Corollary 1. Free semigroups and free commutative semigroups are not fair.

Corollary 2. If $S$ is a right fair semigroup then for every $s \in S$ there exists $n \in \mathbb{N}$ such that $s^{n}$ has a weak right unit. 
Let $U\left(S_{S}\right)$ be the union of all right ideals $I$ of a semigroup $S$ which are right unitary, that is, $I S=I$. Then $U\left(S_{S}\right)$ is the largest right ideal of $S$ which is right unitary. Dually one can consider the left ideal $U\left({ }_{S} S\right)$. Note that if $S$ is a factorisable semigroup then both $S_{S}$ and ${ }_{S} S$ are unitary, hence for factorisable semigroups $S$ we have $U\left(S_{S}\right)=U\left({ }_{S} S\right)=S$.

Corollary 3. For every element $s$ of a right fair semigroup $S$ there exists $n \in \mathbb{N}$ such that $s^{n} \in U\left(S_{S}\right)$.

Proof. Let $s \in S$. Then there exist $n \in \mathbb{N}$ and $u \in S$ such that $s^{n}=s^{n} u$. Now $s^{n}=s^{n} u=s^{n} u u \in\left(s^{n} S^{1}\right) S$ and, for every $v$ in $S, s^{n} v=s^{n} u v \in\left(s^{n} S^{1}\right) S$, so $s^{n} S^{1}$ is a unitary right ideal of $S$. Hence $s^{n} S^{1} \subseteq U\left(S_{S}\right)$ and $s^{n} \in U\left(S_{S}\right)$.

The next lemma will be used repeatedly.

Lemma 1. If $S$ is a right fair semigroup then $U\left(S_{S}\right)$ is a two-sided ideal of $S$.

Proof. By the construction, $U\left(S_{S}\right)$ is a right ideal of $S$. Let $s \in S$ and $u \in U\left(S_{S}\right)$. Since $U\left(S_{S}\right)$ is right unitary, it is right s-unital, so $u=u t$ for some $t \in S$. Hence $s u=s u t$. But then $s u S^{1}=\left(s u S^{1}\right) S$, that is, the principal right ideal $s u S^{1}$ is a unitary right $S$-act. By the definition of $U\left(S_{S}\right), s u \in s u S^{1} \subseteq U\left(S_{S}\right)$. This shows that $U\left(S_{S}\right)$ is a left ideal of $S$.

Lemma 2. Let $S$ be a fair semigroup. For every $s \in S$, the following assertions are equivalent.

(1) $s \in U\left(S_{S}\right)$.

(2) $s=$ su for some $u \in S$.

(3) $s \in U\left({ }_{S} S\right)$.

(4) $s=u s$ for some $u \in S$.

Proof. Since $U\left(S_{S}\right)$ is a right unitary $S$-act, $(1) \Rightarrow(2)$ because $S$ is a fair semigroup. Similarly, $(3) \Rightarrow(4)$.

$(4) \Rightarrow(1)$. Let $s=u s$ for some $u \in S$. By Corollary 3 there exists $n \in \mathbb{N}$ such that $u^{n} \in U\left(S_{S}\right)$. Hence $s=u s=u^{n} s \in U\left(S_{S}\right)$, because $U\left(S_{S}\right)$ is a right ideal. A similar proof works for $(2) \Rightarrow(3)$.

By Lemma 2, $U\left(S_{S}\right)=U\left({ }_{S} S\right)$, so we denote this set by $U(S)$ and call it the unitary part of the fair semigroup $S$.

Corollary 4. If $S$ is a fair semigroup then the set

$$
U(S)=\{s \in S \mid s=s u=v s \text { for some } u, v \in S\}
$$

is a two-sided ideal of $S$. Moreover, $U(S)$ is a semigroup with weak local units (hence also a fair semigroup).

Proof. The first part follows from Lemmas 1 and 2. To prove the second part, take $s \in U(S)$. Then $s=s u=v s$ for some $u, v \in S$. By Corollary 3 , there exist $m, n \in \mathbb{N}$ such that $u^{m}, v^{n} \in U(S)$. Then $s=s u^{m}=v^{n} s$.

Corollary 5. If $S$ is a right fair semigroup, $A_{S}$ is a unitary act and $a \in A$ then $a=$ au for some $u \in U\left(S_{S}\right)$.

Proof. Since $A_{S}$ is unitary, it is s-unital. Hence $a=a s$ for some $s \in S$. By Corollary 3, there exists $n \in \mathbb{N}$ such that $s^{n} \in U\left(S_{S}\right)$. So $a=a s=a s^{n}$. 
We conclude this section by giving several examples of semigroups which are fair and semigroups which are not.

Example 3. 1. Every semigroup with weak local units (in particular every monoid) is a fair semigroup.

2. If $S$ is a semigroup such that $S^{n}$ is a right fair semigroup for some $n \in \mathbb{N}$ then $S$ itself is a right fair semigroup. In particular, every nilpotent semigroup (a semigroup with zero in which every product of a given length is zero) and every infllation of a fair semigroup is a fair semigroup.

3. The multiplicative semigroup of a right xst-ring is a right fair semigroup. To see this, recall first that a module $M_{R}$ over an arbitrary ring $R$ is said to be unitary if $M R=M$ and that every module $M_{R}$ has a largest submodule which is unitary. Let $S$ be a right xst-ring and consider its multiplicative semigroup. Take any sequence $\left(s_{i}\right)_{i \in \mathbb{N}} \in S^{\mathbb{N}}$. In the proof of Proposition 6 of [3] it is shown that there exists $n \in \mathbb{N}$ such that $s_{n} s_{n-1} \ldots s_{1} \in \overline{U\left(S_{S}\right)}$, where $\overline{U\left(S_{S}\right)}$ denotes the largest unitary right ideal of the ring $S$ (we have to distinguish between the largest unitary right ideal of $S$ in the ring sense and in the semigroup sense). By Proposition 1 of [3] this means that the product $s_{n} s_{n-1} \ldots s_{1}$ has a weak right unit. By our Theorem 1, this shows that $S$ is a right fair semigroup.

4. Every finite monogenic semigroup $\langle s\rangle$ is a fair semigroup. If the index of $s$ is at least two then this semigroup is non-factorisable.

5. A homomorphic image of a fair semigroup is a fair semigroup.

6. A direct product of finitely many fair semigroups is a fair semigroup.

Example 4. 1. A subsemigroup of a fair semigroup need not be a fair semigroup: for any semigroup $T, T^{1}$ is a fair semigroup.

2. A direct product of infinitely many fair semigroups is not necessarily a fair semigroup. Let $T_{n}=\left\langle t_{n}\right\rangle, n \in \mathbb{N}$, be an $n$-element monogenic semigroup, where $t_{n}^{n}=t_{n}^{n+1}$. Put $S:=\prod_{n \in \mathbb{N}} T_{n}$ and consider the element $s=\left(t_{1}, t_{2}, t_{3}, \ldots\right) \in S$. If we suppose that $S$ is a fair semigroup then, by Corollary 2 , there exists $k \in \mathbb{N}$ and $u=\left(u_{1}, u_{2}, u_{3}, \ldots\right) \in S$ such that $s^{k} u=s^{k}$. Then $t_{i}^{k} u_{i}=t_{i}^{k}$ for each $i \in \mathbb{N}$. In particular, $t_{k+1}^{k}=t_{k+1}^{k} u_{k+1}=t_{k+1}^{k+1}$, which cannot happen.

3. An ideal extension of a fair semigroup by a fair semigroup need not be itself fair: in Example 2, $I=\{0, a\}$ is an ideal of $S=\{0, a, e\}, S / I$ is a two-element semilattice, hence both of them are fair semigroups by items 1 and 2 in Example 3 , but $S$ is not fair.

4. A finite semigroup generated by two elements need not be fair: $\{a, e\}$ is a generating set for the non-fair semigroup $S=\{0, a, e\}$ in Example 2 .

To give further examples for fair semigroups, we need the following observation.

Lemma 3. If a semigroup $S$ has the descending chain condition (DCC) for principal left ideals then for each sequence $\left(s_{i}\right)_{i \in \mathbb{N}} \in S^{\mathbb{N}}$ there exist $n \in \mathbb{N}$ and $u \in S^{1}$ such that

$$
s_{n} \ldots s_{1}=u s_{n+1} s_{n} \ldots s_{1} .
$$

Proof. Consider a sequence $\left(s_{i}\right)_{i \in \mathbb{N}} \in S^{\mathbb{N}}$ and the descending chain

$$
S^{1} s_{1} \supseteq S^{1} s_{2} s_{1} \supseteq S^{1} s_{3} s_{2} s_{1} \supseteq \ldots
$$

By assumption, there exists $n \in \mathbb{N}$ such that $S^{1} s_{n} \ldots s_{1}=S^{1} s_{n+1} s_{n} \ldots s_{1}$. Hence $s_{n} \ldots s_{1}=u s_{n+1} s_{n} \ldots s_{1}$ for some $u \in S^{1}$. 
Proposition 3. Every commutative semigroup with DCC for principal ideals is a fair semigroup.

Proof. Let $S$ be a commutative semigroup with DCC for principal ideals. Consider a sequence $\left(s_{i}\right)_{i \in \mathbb{N}} \in S^{\mathbb{N}}$. By Lemma 3, there exist $n \in \mathbb{N}$ and $u \in S^{1}$ such that $s_{n} \ldots s_{1}=u s_{n+1} s_{n} \ldots s_{1}$. By commutativity, $s_{n} \ldots s_{1}=\left(s_{n} \ldots s_{1}\right)\left(s_{n+1} u\right)$. Hence $S$ is a fair semigroup by Theorem 1 .

Corollary 6. Every finite commutative semigroup is a fair semigroup.

The converse of Proposition 3 is not true: a commutative monoid need not have DCC for principal ideals.

\section{Morita equivalence}

In this section we study Morita equivalence of right fair semigroups whose unitary part has common weak right local units. We denote the category of all unitary right $S$-acts by UAct ${ }_{S}$. First we define a notion which is analogous to that of a torsionfree module.

Definition 3. A right $S$-act $A_{S}$ is called nonsingular if $a=a^{\prime}\left(a, a^{\prime} \in A\right)$ whenever $a s=a^{\prime} s$ for all $s \in S$.

Proposition 4 (cf. [3], Proposition 2). Let $S$ be a fair semigroup such that $U(S)$ has common weak right local units. Then every unitary right $S$-act is nonsingular.

Proof. Let $A_{S}$ be unitary and let $a, a^{\prime} \in A$ be such that $a s=a^{\prime} s$ for each $s \in S$. By Corollary 5 , there exist $u, u^{\prime} \in U(S)$ such that $a=a u$ and $a^{\prime}=a^{\prime} u^{\prime}$. Let $u v=u$ and $u^{\prime} v=u^{\prime}$ where $v \in U(S)$. Then $a v=a^{\prime} v$ and

$$
a=a u=a u v=a v=a^{\prime} v=a^{\prime} u^{\prime} v=a^{\prime} u^{\prime}=a^{\prime} .
$$

Hence, under the assumptions of Proposition 4, UAct $S$ is the category of unitary nonsingular right $S$-acts.

Definition 4. A right $S$-act $A_{S}$ is called closed if the mapping $\mu_{A}: A \otimes_{S} S \rightarrow$ $A, a \otimes s \mapsto a s$ is bijective.

The category of all closed right $S$-acts is denoted by FAct . $_{\text {. }}$

Obviously, $A_{S}$ is unitary if and only if $\mu_{A}$ is surjective. Hence, for any semigroup $S$, FAct $_{S}$ is a subcategory of UAct . $_{\text {. }}$

Lemma 4. Let $S$ be a semigroup with common weak right local units. Then UAct $_{S}=$ FAct $_{S}$.

Proof. We only need to show that if $A_{S}$ is a unitary act then $\mu_{A}$ is injective. Suppose that $a s=a^{\prime} s^{\prime}, a, a^{\prime} \in A, s, s^{\prime} \in S$. Then there exists $u \in S$ such that $s=s u$ and $s^{\prime}=s^{\prime} u$. Hence $a \otimes s=a \otimes s u=a s \otimes u=a^{\prime} s^{\prime} \otimes u=a^{\prime} \otimes s^{\prime} u=a^{\prime} \otimes s^{\prime}$ in $A \otimes_{S} S$.

Proposition 5 (cf. [3], Proposition 7). Let $S$ be a right fair semigroup such that $I:=U\left(S_{S}\right)$ has common weak right local units. Then

(1) the categories UAct $_{S}$ and $\mathrm{UAct}_{I}$ are isomorphic;

(2) the categories FAct $_{S}$ and FAct $_{I}$ are isomorphic. 
Proof. (1) In a natural way, every right $S$-act is also a right $I$-act. By Corollary 5, every unitary right $S$-act is a unitary right $I$-act. Also, every morphism of right $S$-acts is a morphism of right $I$-acts. In this way we obtain a functor $F:$ UAct $_{S} \rightarrow$ UAct $_{I}$.

Let now $X_{I} \in \mathrm{UAct}_{I}$. We endow the set $X$ with a right $S$-action as follows. Let $x \in X$ and $s \in S$. Since $X_{I}$ is unitary, it is s-unital and hence $x=x u$ for some $u \in I$. Then $u s \in I$, because $I$ is an ideal. We put

$$
x \cdot s:=x(u s) .
$$

Let us check that the definition does not depend on the choice of $u$. Suppose that also $x=x v$ where $v \in I$. By assumption, $u s=u s w$ and $v s=v s w$ for some $w \in I$. Hence $s w \in I$ and

$$
x(u s)=x(u s w)=(x u)(s w)=x(s w)=(x v)(s w)=x(v s w)=x(v s) .
$$

To prove that we obtain an $S$-act, take $x \in X$ and $s, t \in S$. Let $x=x u$ for some $u \in I$. Then $u s=(u s) v$ for some $v \in I$. So $x(u s)=x((u s) v)$ and

$$
\begin{aligned}
(x \cdot s) \cdot t & =x(u s) \cdot t=(x(u s))(v t)=x((u s)(v t))=x(((u s) v) t)=x((u s) t) \\
& =x(u(s t))=x \cdot(s t) .
\end{aligned}
$$

Suppose now that $g: X_{I} \rightarrow Y_{I}$ is a morphism of unitary right $I$-acts. Let $x \in X$, $s \in S$, and let $u \in I$ be such that $x=x u$. Then $g(x)=g(x) u$ and

$$
g(x \cdot s)=g(x(u s))=g(x)(u s)=g(x) \cdot s,
$$

which means that $g$ is a morphism of right $S$-acts. So putting $G\left(X_{I}\right):=X_{S}$ and $G(g):=g$ we obtain a functor $G: \mathrm{UAct}_{I} \rightarrow$ UAct $_{S}$.

Let us prove that $G\left(F\left(A_{S}\right)\right)=A_{S}$, that is, that the $S$-action of $G\left(F\left(A_{S}\right)\right)$ coincides with the $S$-action of $A_{S}$. Take $a \in A, s \in S$ and let $a=a u$ where $u \in I$. Then the $S$-action of $G\left(F\left(A_{S}\right)\right)$ is $(a, s) \mapsto a(u s)=(a u) s=a s$, as needed.

Finally we show that $F\left(G\left(X_{I}\right)\right)=X_{I}$. Consider a unitary $I$-act $X_{I}$ with the $I$-action $(x, u) \mapsto x * u$. Take $x \in X$ and $v \in I$. Let $u \in I$ be such that $x=x * u$. Then the $I$-action of $F\left(G\left(X_{I}\right)\right)$ is $(x, v) \mapsto x *(u v)=(x * u) * v=x * v$, that is, the same as the $I$-action of $X_{I}$. This completes the proof of the first part.

(2) a) First we show that $F$ takes closed right $S$-acts to closed right $I$-acts. Because of the remark after Definition 4, we only need to consider injectivity of the corresponding $\mu$. Assume that $A_{S}$ is closed and consider the mapping $\mu: A \otimes_{I} I \rightarrow$ $A, a \otimes u \mapsto a u$. Suppose that $a u=a^{\prime} u^{\prime}$ where $a, a^{\prime} \in A$ and $u, u^{\prime} \in I$. We need to prove that $a \otimes u=a^{\prime} \otimes u^{\prime}$ in $A \otimes_{I} I$. By assumption, $a \otimes u=a^{\prime} \otimes u^{\prime}$ in $A \otimes_{S} S$. Hence

$$
\begin{aligned}
& a=a_{1} s_{1} \quad s_{1} u=t_{1} z_{1} \\
& a_{1} t_{1}=a_{2} s_{2} \quad s_{2} z_{1}=t_{2} z_{2} \\
& a_{2} t_{2}=a_{3} s_{3} \quad s_{3} z_{2}=t_{3} z_{3} \\
& \begin{aligned}
& \cdots \\
a_{n-1} t_{n-1} & =a_{n} s_{n} \quad s_{n} z_{n-1} \quad \cdots \\
a_{n} t_{n} & =a^{\prime}
\end{aligned}
\end{aligned}
$$

for some $a_{k} \in A, s_{k}, t_{k} \in S^{1}, z_{l} \in S$, where $k \in\{1, \ldots, n\}$ and $l \in\{1, \ldots, n-1\}$. For each $k$ there exists $u_{k} \in I$ such that $a_{k}=a_{k} u_{k}$. Now $u_{k} t_{k} z_{k} \in I$, and since every finite subset of $I$ has a common weak right local unit, there exists $v \in I$ such 
that $u_{k} t_{k} z_{k}=u_{k} t_{k} z_{k} v$ for each $k$. Therefore

$$
\begin{aligned}
a \otimes u & =a_{1} u_{1} s_{1} \otimes u=a_{1} \otimes u_{1} s_{1} u=a_{1} \otimes u_{1} t_{1} z_{1}=a_{1} \otimes u_{1} t_{1} z_{1} v \\
& =a_{1} u_{1} t_{1} \otimes z_{1} v=a_{2} u_{2} s_{2} \otimes z_{1} v=a_{2} \otimes u_{2} s_{2} z_{1} v \\
& =a_{2} \otimes u_{2} t_{2} z_{2} v=\ldots=a^{\prime} \otimes u^{\prime}
\end{aligned}
$$

in $A \otimes_{I} I$, as needed.

b) We prove that $G$ takes closed right $I$-acts to closed right $S$-acts. Consider a closed $I$-act $X_{I}$. Take $x, x^{\prime} \in X$ and $s, s^{\prime} \in S$. Let $u, u^{\prime}$ be such that $x=x u$ and $x^{\prime}=x^{\prime} u^{\prime}$. Suppose that $x \cdot s=x^{\prime} \cdot s^{\prime}$, that is, $x(u s)=x^{\prime}\left(u^{\prime} s^{\prime}\right)$. By assumption, $x \otimes u s=x^{\prime} \otimes u^{\prime} s^{\prime}$ in $X \otimes_{I} I$. But then obviously $x \otimes u s=x^{\prime} \otimes u^{\prime} s^{\prime}$ in $X \otimes_{S} S$ and hence $x \otimes s=x^{\prime} \otimes s^{\prime}$ in $X \otimes_{S} S$. So the mapping $\mu: X \otimes_{S} S \rightarrow X, x \otimes s \mapsto x \cdot s$ is injective and $X_{S}$ is closed.

In the ring case (see [3], Proposition 7) no assumption is needed on $U\left(S_{S}\right)$, but here we cannot do without assuming the existence of common weak right units in it. Namely, in the ring case this condition follows from the s-unital property by a simple orthogonalisation (see [10], Theorem 1), but in semigroups it is obviously not true.

Definition 5. Semigroups $S$ and $T$ are called right Morita equivalent if the categories FAct $_{S}$ and FAct $_{T}$ are equivalent, left Morita equivalent if the categories ${ }_{S}$ FAct and ${ }_{T}$ FAct are equivalent, Morita equivalent if they are left and right Morita equivalent, and strongly Morita equivalent (cf. [9]) if there exists a unitary Morita context $\left(S, T,{ }_{S} P_{T},{ }_{T} Q_{S}, \theta, \phi\right)$ with $\theta$ and $\phi$ surjective.

By Proposition 1 of [6] we know that if two semigroups are strongly Morita equivalent then both of them must be factorisable.

In the papers [6], [8], and [9] one can find many examples of (strongly) Morita equivalent semigroups with local units. Next we give some examples in the case of semigroups which do not have local units.

Example 5. Consider a non-trivial semigroup $S$ with zero multiplication. Then $S$ is a fair semigroup where $U(S)=\{0\}$ has common weak local units. By Proposition 5 and its dual, $S$ is right and left Morita equivalent to a one-element semigroup. But a non-trivial semigroup with zero multiplication is not factorisable, hence it cannot be strongly Morita equivalent to any semigroup.

Consider now Example 2 again: let $Z$ be a semigroup with zero multiplication, and extend $Z$ with a right identity $e$ such that $e z=0$ for all $z \in Z$. As we have seen in Example 2, $S$ is factorisable and right fair but not left fair. We claim that $S$ is strongly Morita equivalent to the two-element semilattice. Indeed, by Theorem 9 of [8], a semigroup $S$ is strongly Morita equivalent to a monoid if and only if $S=S e S$ for some idempotent $e \in S$. In our case we have $s=$ see for every $s \in S$, hence $S=S e S$. From the proof of Theorem 9 in [8] it follows that $S$ is strongly Morita equivalent to the two-element monoid $e S e=\{e, 0\}$.

Proposition 6. Every finite monogenic semigroup is Morita equivalent to its group part. Consequently, two such semigroups are Morita equivalent if and only if their periods are equal. 
Proof. If $S$ is a finite monogenic semigroup then $U(S)$ is the group part of $S$, and the first claim is true by Proposition 5. By [1] and [4], two groups are Morita equivalent if and only if they are isomorphic, which gives the second claim.

Example 6. Suppose that $S=\langle s\rangle$ is a finite monogenic semigroup where the index of $s$ is $\geq 2$ and the group part is $T$. Then $S$ and $T$ are left and right Morita equivalent but they are not strongly Morita equivalent since $S$ is not factorisable (the element $s$ has no factorisation).

Let us also mention that Banaschewski [1] and Knauer [4] showed that if two monoids $S$ and $T$ are Morita equivalent and $S$ is a group or is commutative then $S$ and $T$ are isomorphic. The proposition above shows that the situation is different in the case of semigroups: a (finite) abelian group can be Morita equivalent to a semigroup which is not even factorisable.

Theorem 2. Let $S, T$ be right fair semigroups such that $U\left(S_{S}\right), U\left(T_{T}\right)$ have common weak right local units. Then the following are equivalent.

(1) The categories UAct $_{S}$ and $\mathrm{UAct}_{T}$ are equivalent.

(2) The categories FAct $_{S}$ and FAct $_{T}$ are equivalent.

(3) The semigroups $S$ and $T$ are Morita equivalent.

(4) The categories $\mathrm{UAct}_{U\left(S_{S}\right)}$ and $\mathrm{UAct}_{U\left(T_{T}\right)}$ are equivalent.

(5) The categories $\mathrm{FAct}_{U\left(S_{S}\right)}$ and $\mathrm{FAct}_{U\left(T_{T}\right)}$ are equivalent.

(6) The semigroups $U\left(S_{S}\right)$ and $U\left(T_{T}\right)$ are Morita equivalent.

Proof. (1) $\Leftrightarrow(4)$ and $(2) \Leftrightarrow(5)$ by Proposition 5 . (4) $\Leftrightarrow$ (5) by Lemma 4 . (2) $\Leftrightarrow$ (3) and $(5) \Leftrightarrow(6)$ by Definition 5 .

Theorem 2 reduces the study of Morita equivalence of fair semigroups $S, T$ whose unitary parts $U(S), U(T)$ have common weak right local units to the study of Morita equivalence of their unitary parts. Since $U(S), U(T)$ are factorisable semigroups (actually semigroups with weak local units), results from [2] concerning unitary acts can be applied. If it happens that $U(S), U(T)$ are semigroups with local units then also the theory developed in [8] and [6] is applicable.

For a semigroup $S$, define the congruence

$$
\zeta_{S}=\left\{\left(s_{1}, s_{2}\right) \in S \times S \mid s s_{1}=s s_{2} \text { for all } s \in S\right\}
$$

and denote by $S^{\prime}$ the quotient semigroup $S / \zeta_{S}$. From [2], Theorem 3 we have the following result.

Proposition 7. Let $S$ and $T$ be factorisable semigroups. The category of unitary nonsingular right $S$-acts is equivalent to the category of unitary nonsingular right $T$-acts if and only if the semigroups $S^{\prime}$ and $T^{\prime}$ are strongly Morita equivalent.

For left reductive semigroups, $\zeta_{S}$ is the equality relation and $S^{\prime}=S$. Now, if $U(S), U(T)$ have common weak local units then they are left reductive, hence $U(S)^{\prime}=U(S)$ and $U(T)^{\prime}=U(T)$.

Proposition 8. Let $S, T$ be fair semigroups such that $U(S), U(T)$ have common weak local units. Then the following are equivalent.

(1) The semigroups $S$ and $T$ are Morita equivalent.

(2) The semigroups $U(S)$ and $U(T)$ are Morita equivalent.

(3) The semigroups $U(S)$ and $U(T)$ are strongly Morita equivalent. 
Proof. The equivalence of (1) and (2) follows immediately from Theorem 2. By the same theorem, $U(S)$ and $U(T)$ are Morita equivalent if and only if $\operatorname{UAct}_{U(S)}$ and $\mathrm{UAct}_{U(T)}$ are equivalent. By Proposition 4, UAct $)_{U(S)}\left(\mathrm{UAct}_{U(T)}\right)$ is the category of unitary nonsingular right $U(S)$-acts $(U(T)$-acts). It follows from Proposition 7 that the last two categories are equivalent if and only if $U(S)$ and $U(T)$ are strongly Morita equivalent. This completes the proof of the equivalence of (2) and (3).

Corollary 7. Two semigroups with common weak local units are Morita equivalent if and only if they are strongly Morita equivalent.

Proof. By Proposition 2, a semigroup has weak local units if and only if it is factorisable and fair, and for a factorisable semigroup $S$ we have $U(S)=S$, and now our claim follows from the equivalence of the last two conditions of the foregoing proposition.

Concluding remarks. 1. For semigroups with local units, left Morita equivalence, right Morita equivalence, and strong Morita equivalence are the same relation by [8]. It is not known how much of this remains true for semigroups which do not have local units. We do not have an answer even for semigroups with weak local units or for factorisable semigroups. All we know is that Morita equivalence and strong Morita equivalence do not coincide in the class of all semigroups.

2. In [7], several properties are shown to be invariant under strong Morita equivalence in the presence of factorisability or various kinds of local units. Example 7 shows that the properties of being a group, being a monoid, having local units or weak local units, and factorisability are not invariant under Morita equivalence in the class of all semigroups. In Example 5 we have seen that every semigroup with zero multiplication is Morita equivalent to the one-element semigroup, and by Theorem 16 in [6] we know that a semigroup is strongly Morita equivalent to a one-element semigroup if and only if it is a rectangular band. Hence being a rectangular band is also not invariant under Morita equivalence. These examples show also that the ideal lattice of a semigroup need not be preserved under Morita equivalence.

\section{ACKNOWLEDGEMENT}

We are grateful to Iiris Lüsi for finding Example 2. We would also like to thank Victoria Gould for discussions that led us to Proposition 3.

\section{REFERENCES}

[1] B. Banaschewski, Functors into categories of $M$-sets, Abh. Math. Sem. Univ. Hamburg 8 (1972), 49-64.

[2] Y. Q. Chen, K. P. Shum, Morita equivalence for factorisable semigroups, Acta Math. Sin. (Engl. Ser.) 17 (2001), 437-454.

[3] J.L. García, L. Marín, Rings having a Morita-like equivalence, Commun. Algebra 27 (1999), 665-680.

[4] U. Knauer, Projectivity of acts and Morita equivalence of monoids, Semigroup Forum 3 (1972), 359-370.

[5] V. Laan, Acceptable Morita contexts for semigroups, ISRN Algebra, vol. 2012, article ID 725627, 2012, 5 pp.

[6] V. Laan, L. Márki, Strong Morita equivalence of semigroups with local units, J. Pure Appl. Algebra 215 (2011), 2538-2546.

[7] V. Laan, L. Márki, Morita invariants for semigroups with local units, Mh. Math. 166 (2012), 441-451. 
[8] M.V. Lawson, Morita equivalence of semigroups with local units, J. Pure Appl. Algebra 215 (2011), 455-470.

[9] S. Talwar, Strong Morita equivalence and a generalisation of the Rees theorem, J. Algebra 181 (1996), 371-394

[10] H. Tominaga, On s-unital rings, Math. J. Okayama Univ. 18 (1976), 117-134.

[11] Y. Xu, K.P. Shum, R.F. Turner-Smith, Morita-like equivalence of infinite matrix subrings, J. Algebra 159 (1993), 425-435.

Institute of Mathematics, Faculty of Mathematics and Computer Science, J. Livi 2, University of Tartu, 50409 Tartu, Estonia

E-mail address: vlaan@ut.ee

Alfréd Rényi Institute of Mathematics, Hungarian Academy of Sciences, H-1364 BuDAPeSt, Pf. 127, Hungary

E-mail address: marki.laszlo@renyi.mta.hu 